\title{
Role of DNA damage-induced replication checkpoint in promoting lesion bypass by translesion synthesis in yeast
}

\author{
Vincent Pagès, Sergio R. Santa Maria, Louise Prakash, ${ }^{1}$ and Satya Prakash \\ Department of Biochemistry and Molecular Biology, University of Texas Medical Branch at Galveston, Galveston, \\ Texas 77555, USA
}

Unrepaired DNA lesions in the template strand block the replication fork. In yeast, Mec1 protein kinase-mediated replication checkpoint prevents the breakdown of replication forks and maintains viability in DNA-damaged cells going through the $S$ phase. By ensuring that the replisome does not dissociate from the fork stalled at the lesion site, the replication checkpoint presumably coordinates the action of lesion bypass processes with the replisome. However, it has remained unclear as to which of the lesion bypass processes-translesion synthesis (TLS) and/or template switching-depend on the activation of the replication checkpoint. Here we determine if the Mec1 kinase and the subunits of the checkpoint clamp and the clamp loader are required for TLS. We show that proficient TLS can occur in the absence of these checkpoint proteins in nucleotide excision repair (NER)-proficient cells; however, in the absence of NER, checkpoint protein-mediated Rev1 phosphorylation contributes to increasing the proficiency of DNA polymerase $\zeta$-dependent TLS.

[Keywords: DNA damage; translesion synthesis; replication checkpoint; lesion bypass; Mec1 kinase Rev1 phosphorylation]

Supplemental material is available at http://www.genesdev.org.

Received February 20, 2009; revised version accepted May 5, 2009.

Unrepaired DNA lesions in the template strand block synthesis by replicative DNA polymerases. In eukaryotes, the Rad6-Rad18 ubiquitin-conjugating enzyme complex regulates replication through DNA lesions via translesion synthesis (TLS) by specialized DNA polymerases (Pols) and by means that use template switching (Prakash et al. 2005). Genetic studies in the yeast Saccharomyces cerevisiae have indicated that Rad6Rad18-dependent replication through UV-induced DNA lesions can occur by Poln- or Pol $\zeta$-mediated TLS (Nelson et al. 1996b; Johnson et al. 1999, 2000a; Bresson and Fuchs 2002; Prakash et al. 2005), or by a Rad5-Mms2-Ubc13 pathway that promotes lesion bypass by template switching (Torres-Ramos et al. 2002; Gangavarapu et al. 2006; Blastyak et al. 2007). A Rad6-Rad18-independent template-switching pathway dependent on Rad51, Rad52, and Rad54 proteins can also restore the continuity of newly synthesized DNA from UV-damaged DNA templates (Prakash 1981; Gangavarapu et al. 2007).

When progression of the replication fork is halted by a DNA lesion or by inhibitors of DNA synthesis, eukaryotic cells activate an intra-S-phase checkpoint pathway,

${ }^{1}$ Corresponding author.

E-MAIL 1.prakash@utmb.edu; FAX (409) 747-8608.

Article is online at http://www.genesdev.org/cgi/doi/10.1101/gad.1793409. also known as a replication checkpoint (Paulsen and Cimprich 2007). The activated replication checkpoint plays a crucial role in the stabilization of replication forks by maintaining the association of the replisome with the fork, thereby enabling resumption of replication from the stalled fork after the lesion has been bypassed (Tercero and Diffley 2001; Tercero et al. 2003; Paulsen and Cimprich 2007). In the absence of a replication checkpoint, the replisome dissociates and the stalled replication forks degenerate, generating large sections of ssDNA and the accumulation of reversed forks (Sogo et al. 2002; Cobb et al. 2003, 2005; Paulsen and Cimprich 2007). The replication checkpoint also slows S-phase progression, allowing time for DNA repair or lesion bypass to occur, and down-regulates late origin firing (Tercero et al. 2003; Paulsen and Cimprich 2007).

In S. cerevisiae, Mec1 kinase is a key component of the replication checkpoint, and it initiates the checkpoint pathway by phosphorylating the downstream effector kinase Rad53 (Nyberg et al. 2002; Paulsen and Cimprich 2007). Checkpoint activation by Mecl additionally may require the checkpoint clamp-clamp loader complex, in which the checkpoint clamp is a heterotrimer comprised of Rad17, Mec3 (we find that MEC3 and RAD15 represent the same gene), and Ddc1 subunits, and the clamp loader $\operatorname{Rad} 24-\mathrm{Rfc}$ is comprised of the four small subunits of Rfc 
(Rfc2-5) together with the Rad24 subunit. In vitro studies with purified checkpoint proteins have shown that the checkpoint clamp loaded onto partial duplex DNA activates the kinase activity of Mecl and the activated Mec1, then phosphorylates Rad53 (Majka and Burgers 2003).

Although experimental evidence clearly points to the requirement of a Mec1-controlled replication checkpoint for the stabilization of replication forks in DNA-damaged yeast cells, it remains unclear as to which of the lesion bypass processes-TLS or template switching-requires activation of the replication checkpoint. For example, one could envisage a situation in which a lesion bypass process operates in such tight coordination with the DNA replication ensemble that progression of the replication fork is halted minimally. In that case, the absence of multiple stalled replication forks might fail to generate the threshold level of signal necessary for checkpoint activation to occur (Tercero et al. 2003).

The most pertinent information relating to the requirement of checkpoint proteins in lesion bypass comes from the study of Hartwell and colleagues (Paulovich et al. 1998), in which they showed that the frequency of UV-induced mutations shows a considerable reduction in a nucleotide excision repair (NER)-defective yeast strain in the absence of checkpoint clamp and clamp loader proteins. Since UV-induced mutations result from TLS mediated by Pol $\zeta$ opposite cyclobutane pyrimidine dimers (CPDs) and (6-4) photoproducts, one possible interpretation of these observations is that these checkpoint proteins help maintain a stably paused replication fork at UV lesions for TLS to occur.

In this study, we investigate the role of the Mec1dependent replication checkpoint in TLS in UV-damaged yeast cells. We find that in wild-type yeast cells, proficient TLS occurs in the absence of Mec1 kinase or the components of checkpoint clamp and clamp loader. In NER-defective cells, however, the proficiency of Pol $\zeta$ mediated TLS is reduced in the absence of these checkpoint proteins. To decipher how checkpoint proteins modulate TLS proficiency in NER-deficient cells, we analyzed the impact that Rev1 phosphorylation, which we show to be dependent on these checkpoint proteins, has on the proficiency of Polל-mediated TLS. We conclude that although the replication checkpoint is not essential for TLS to occur, checkpoint protein-mediated Rev1 phosphorylation contributes to increasing the proficiency of Pol $\zeta$-mediated TLS, the need for which becomes evident in the absence of NER.

\section{Results}

Mec1 kinase is dispensable for UV mutagenesis in wild-type yeast cells

Since (6-4) photoproducts are removed much more rapidly by NER than are CPDs, UV mutations in wild-type yeast result predominantly from TLS opposite CPDs (Sancar 1996; You et al. 2001). In yeast, Pol $\eta$ and Polל promote TLS opposite CPDs, wherein Pol $\eta$ acts primarily in an error-free manner and Polל functions in a more mutagenic manner. Whereas Pol $\eta$ can carry out proficient and relatively error-free TLS through CPDs (Johnson et al. 1999, 2000b; Washington et al. 2000, 2003), Polל contributes to their bypass by extending from the correct or incorrect nucleotide inserted opposite the $3^{\prime}$ pyrimidine of the CPD by another Pol (Johnson et al. 2000a).

To determine whether activation of the replication checkpoint is required for TLS, we analyzed the frequency of UV-induced $C A N 1^{S}$ to $c a n 1^{r}$ forward mutations in yeast cells carrying deletion mutations of the various checkpoint genes. Since Mecl kinase is the initiator of replication checkpoint and is indispensable for maintaining replication fork stability in DNA-damaged yeast cells, we first examined whether Mec1 was required for UV mutagenesis in wild-type cells. However, because $M E C 1$ is essential for cell viability, but viability can be restored to mec1 $1 \Delta$ cells by the sml1s mutation, we examined UV mutagenesis in the sml1s and sml1s mec1s strains. UV sensitivity or UV mutagenesis is not affected by the sml1s mutation (cf. Figs. 1 and 2). The mec1s mutation confers an increase in UV sensitivity, but the frequency of UV-induced mutations remains about the same in the sml1s mec1s strain as in the sml1s strain (Fig. 1). Since UV-induced mutations in NER-proficient cells result predominantly from Polל action opposite CPDs and, to a much lesser extent, opposite (6-4) photoproducts, this result indicates that

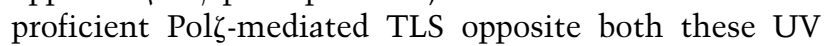
lesions can operate in the absence of Mec1. To verify that UV mutations in the sml1s mec1s strain arise from Polל action, we examined UV-induced mutation frequencies in the sml1s mec1s rev3s strain and, as expected, the incidence of UV mutagenesis was reduced in this strain to the same extent as in the rev3s strain (Fig. 1). Because of the role of Poln in error-free TLS opposite CPDs, the frequency of UV-induced mutations rises in the rad304 strain, which lacks Poln (McDonald et al. 1997; Yu et al. 2001). The incorporation of the rad304 mutation into the sml1s mec1s strain led to an increase in the UV sensitivity of the latter strain, and the frequency of UVinduced mutations was enhanced in the sml1s mec1s

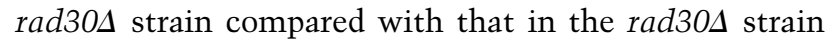
(Fig. 1). The increase in UV mutagenesis is particularly evident at the higher UV dose examined $\left(10 \mathrm{~J} / \mathrm{m}^{2}\right)$, where we observe an approximately fourfold increase in mutation frequencies in the sml1s mec1s rad $30 \Delta$ strain over that in the rad30د strain. Since the absence of Poln from the sml1s mec1s strain leads to an enhancement of UV sensitivity and confers an increase in the frequency of UV-induced mutations, these observations imply that Pol $\eta$ is able to function in TLS in the absence of Mec1.

In yeast cells treated with DNA-damaging agents, PCNA is monoubiquitinated at its Lys-164 residue by the Rad6-Rad18 enzyme complex, and genetic studies in yeast have shown that PCNA monoubiquitination is essential for TLS (Hoege et al. 2002; Stelter and Ulrich 2003; Haracska et al. 2004). The Lys-164-linked ubiquitin moiety is subsequently modified by Lys-63-linked polyubiquitination, which additionally requires the Mms2Ubc13 ubiquitin-conjugating enzyme complex together 
Pagès et al.
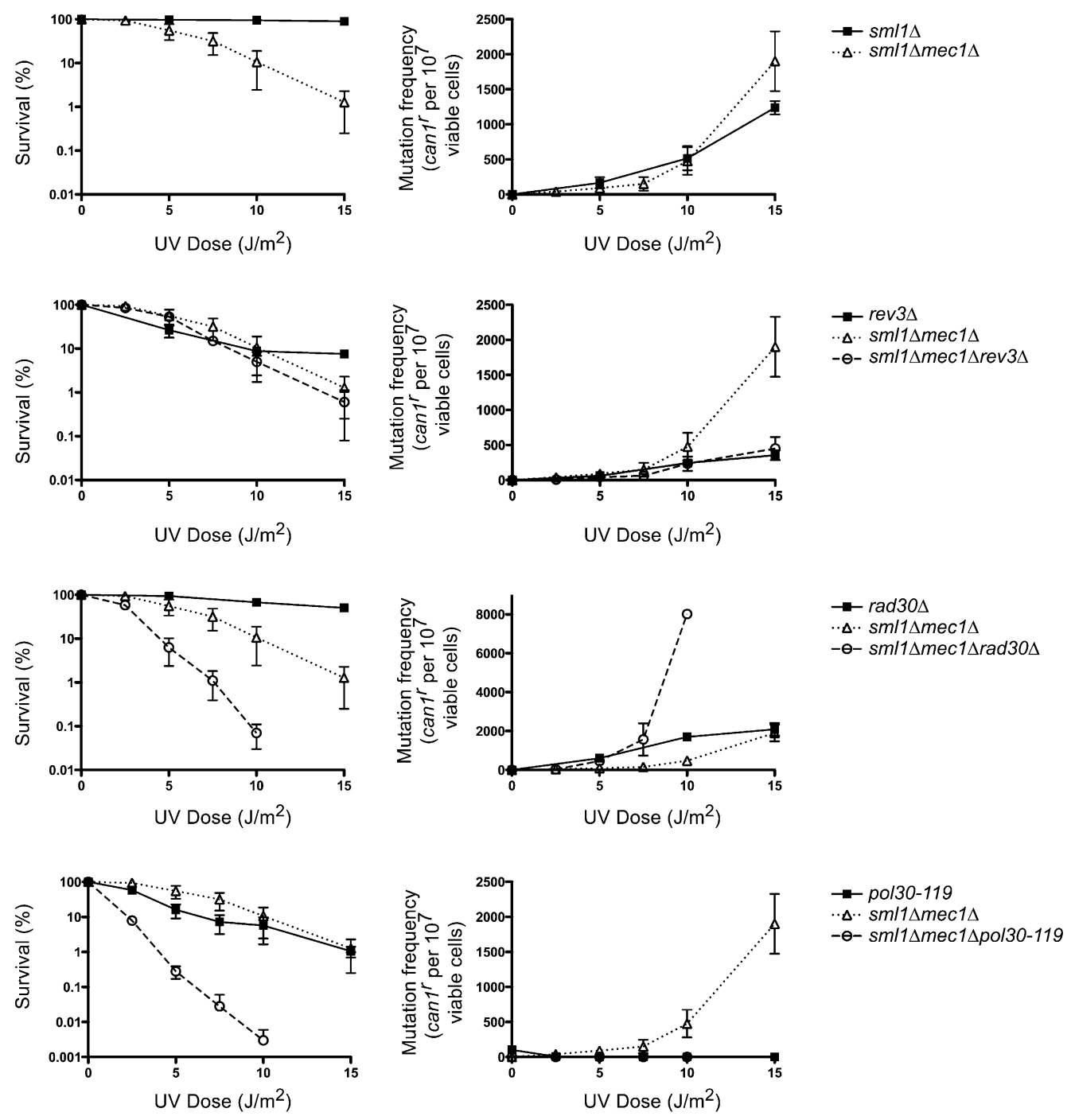

Figure 1. Mec1 is not required for UV mutagenesis. UV survival and the frequencies of UV-induced $\operatorname{can} 1^{r}$ mutations were determined

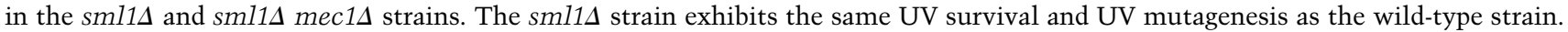

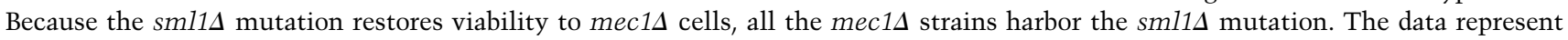
averages of three independent experiments and error bars represent standard deviations of determinations. In this figure and Figures 2, 5, and 6, the apparent absence of error bars in some cases is because the error bars are too small.

with Rad5, which acts as the ubiquitin ligase in the Mms2-Ubc13-Rad5 complex (Hoege et al. 2002). Since PCNA monoubiquitination is required for TLS and PCNA polyubiquitination is required for template switching mediated by the helicase function of Rad5, both of these Rad6-Rad18 lesion bypass pathways are inhibited by the pol30-119 mutation wherein the Lys-164 residue of PCNA has been changed to arginine, and therefore cannot be ubiquitinated (Haracska et al. 2004). To establish that UV mutations in the mec1s strain arise from TLS and not from a role of Pol $\zeta$ in some other as-yetundefined pathway, we determined whether UV-induced mutations that arise in the mec1s strain require PCNA ubiquitination. As shown in Figure 1, a drastic reduction in UV mutagenesis occurs in the sml1s mec1s pol30-119 strain similar to that in the pol30-119 strain, indicating that UV-induced mutations in the mec1s strain arise from PCNA ubiquitination-dependent Pol $\zeta$-mediated TLS.

Although Rad6-Rad18-dependent lesion bypass by TLS or by template switching does not occur in the pol30-119 mutant, this mutation does not impart the same high degree of UV sensitivity as the rad64 or rad184 mutations. This is because the Lys-164 residue of PCNA also undergoes the SUMO modification in DNA-damaged yeast cells (Hoege et al. 2002) and, in the absence of this modification, the Rad52-dependent lesion bypass pathway becomes activated (Haracska et al. 2004). Our observation that the sml1s mec1s pol30-119 mutant exhibits a large synergistic enhancement of UV sensitivity compared with the UV sensitivity for the sml1s mec1s and pol30-119 mutants raises the possibility that 

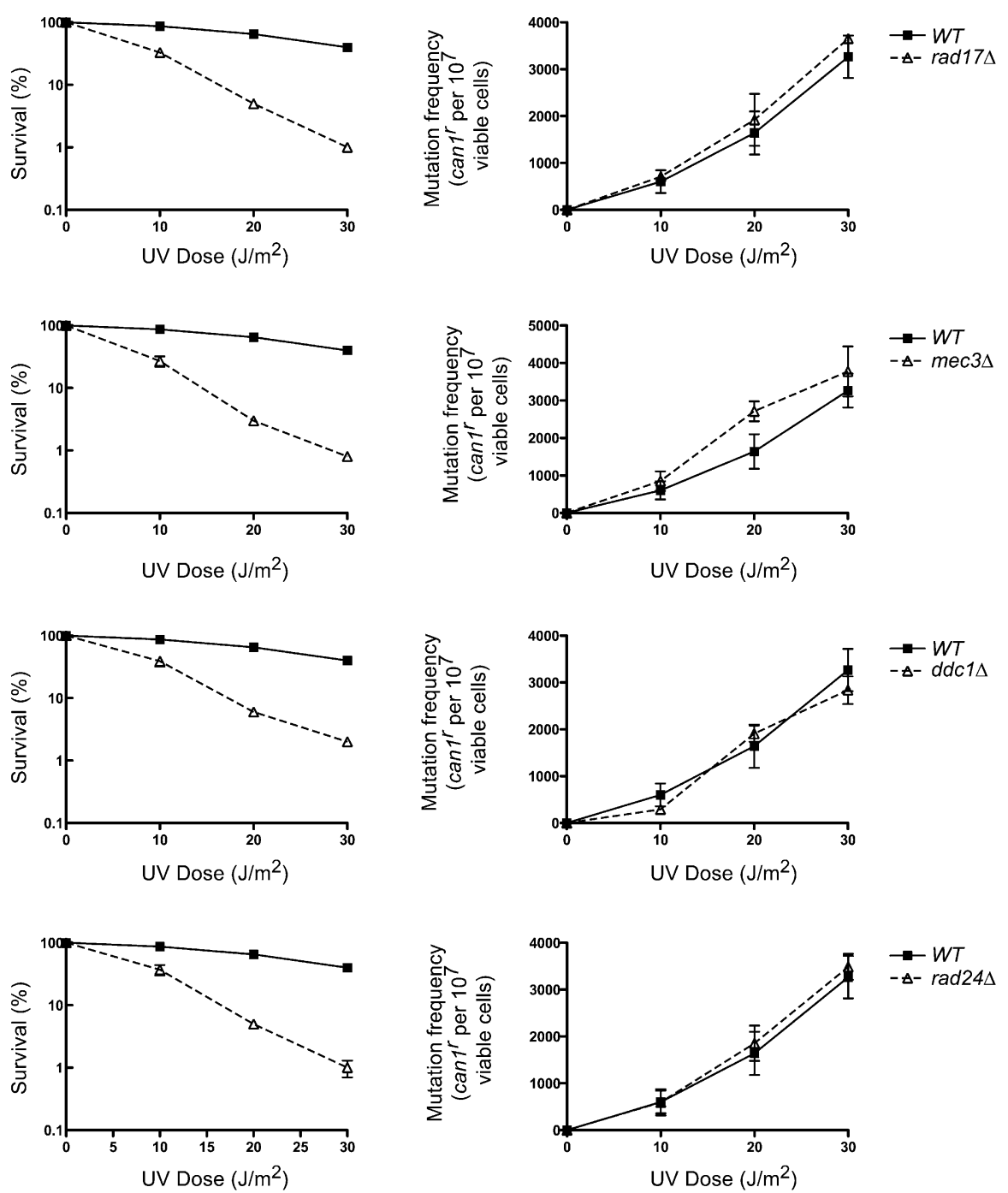

Figure 2. The Rad17, Mec3, and Ddc1 subunits of checkpoint clamp and Rad24 subunit of checkpoint clamp loader are not required for UV mutagenesis. UV survival and the frequencies of UV-induced can $1^{r}$ mutations were determined in the wild-type and isogenic

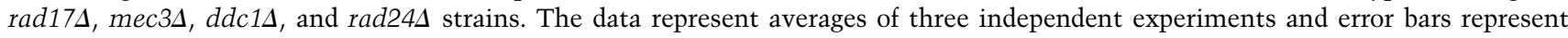
standard deviations.

the Mec1-dependent checkpoint pathway plays an important role in promoting lesion bypass by the Rad52dependent recombination pathway.

\section{Checkpoint clamp and clamp loader are not required for UV mutagenesis in wild-type yeast cells}

To determine whether the checkpoint clamp and clamp loader proteins were also dispensable for TLS, we examined the frequency of UV-induced can $1^{T}$ mutations in

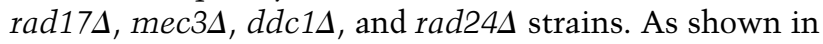
Figure 2, although all of these checkpoint mutations confer an increase in UV sensitivity, they have no effect on UV mutagenesis. Thus, our data show that neither the Mec1 kinase nor the various components of checkpoint clamp and clamp loader are required for proficient TLS in yeast cells.
Rev1 phosphorylation requires the Mec1-Ddc2 kinase and the components of check point clamp and clamp loader

Rev1, a Y family DNA Pol, physically associates with Pol $\zeta$ and plays an indispensable structural role in Pol $\zeta$ mediated TLS (Acharya et al. 2006). Since Rev1 has been shown recently to be phosphorylated in response to DNA damage, and since this phosphorylation requires $\mathrm{Mecl}$ (Sabbioneda et al. 2007), we considered the possibility that Rev1 phosphorylation might contribute to increasing the proficiency of Pol $\zeta$-mediated TLS, the need for which may become more acute when the lesion load rises, as in the absence of NER. Furthermore, we speculated that if Rev1 phosphorylation were found to also require the checkpoint clamp and clamp loader, then that might provide a rationale for the reduction in UV 
mutagenesis that has been reported to occur in the absence of these checkpoint proteins in an NER-defective yeast strain (Paulovich et al. 1998).

To determine if the checkpoint clamp and clamp loader affect an increase in the proficiency of Pol $\zeta$-mediated TLS via their role in Rev1 phosphorylation, we first examined the status of Rev1 phosphorylation in the absence of these checkpoint proteins following treatment with 4NQO. Rev1 phosphorylation is induced in wild-type yeast cells following treatment with 4NQO (Fig. 3A). As has been reported previously, damage-inducible Rev1 phosphorylation is absent in the mec1s strain (Sabbioneda et al. 2007). Since Ddc2 is the nonkinase subunit of Mec1 and is essential for Mecl function in checkpoint activation, we examined if Ddc2 was also required for Rev1 phosphorylation. As expected, we find that Rev1 phosphorylation does not occur in the 4NQO-treated $d d c 2 \Delta$ strain (Fig. 3A). In contrast to the requirement of Mec1Ddc2 for Rev1 phosphorylation, however, efficient Rev1 phosphorylation was found to occur in rad534 cells following 4NQO treatment (Fig. 3A).

Next, we examined if the components of checkpoint clamp, Rad17, Mec3, and Ddc1, were required for damage-induced Rev1 phosphorylation. As shown in Figure $3 \mathrm{~B}$, we found no evidence of Rev1 phosphorylation in the absence of any of these proteins. Also, our results show that $\operatorname{Rad} 24$, which represents the largest subunit specific for checkpoint clamp loader, is required for Rev1 phosphorylation. In summary, damage-inducible Rev1 phosphorylation requires the Mec1-Ddc2 kinase complex and the components of checkpoint clamp and clamp loader, but not the Rad53 kinase.

A.

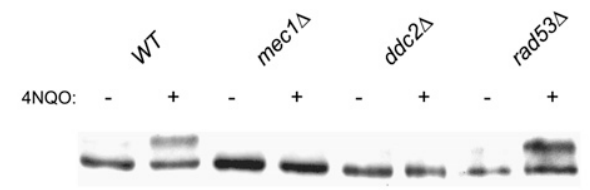

B.

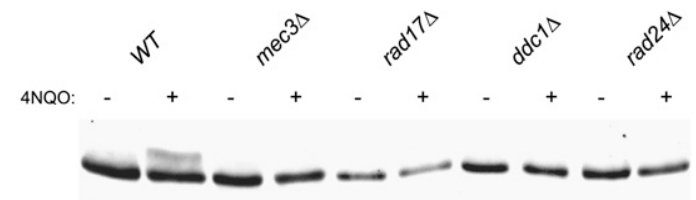

Figure 3. Requirement of checkpoint proteins for DNA damageinduced Rev1 phosphorylation. The Rev1 protein tagged at the C terminus with 4HA was expressed from plasmid pVP64 in the wild-type or the checkpoint mutant strain. Exponentially growing cells were treated $(+)$ or not $(-)$ with 4NQO $(2 \mu \mathrm{g} / \mathrm{mL})$ for $15 \mathrm{~min}$. Cells were then washed with water and total protein extract was obtained in the presence of protease inhibitors and phosphatase inhibitors. Protein extracts were analyzed on $6 \%$ acrylamide gels (77:1 acrylamide:bis-acrylamide), and Rev1 was detected by Western blotting with anti-HA antibody using standard techniques. (A) Rev1 phosphorylation is inhibited in the absence of Mec1-Ddc2 kinase but not in the absence of Rad53 kinase. (B) Rev1 phosphorylation does not occur in

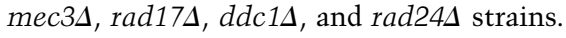

Rev1 phosphorylation modulates the efficiency of Polל-mediated TLS in NER-defective cells

To determine the contribution that Rev1 phosphorylation makes to Pol $\zeta$-mediated TLS, we sought to identify the phosphorylation site in Rev1 so that the effects of loss of phosphorylation could then be assessed by mutational studies. Rev1 has seven consensus $(\mathrm{S} / \mathrm{T}) \mathrm{Q}$ sites that are the potential targets of Mecl kinase (Fig. 4A). We individually changed each of these serine or threonine residues to an alanine and examined the phosphorylation status of Revl protein encoded by each of these mutant alleles. As shown in Figure 4B, 4NQO-inducible Rev1 phosphorylation does not occur when the Ser-31 residue present in the SQ1 site is changed to alanine, whereas mutational inactivation of the remaining six SQ/TQ sites has no effect on Rev1 phosphorylation.

Next, we examined the effects of mutational inactivation of Rev1 phosphorylation on UV sensitivity and on UV-induced $\operatorname{can}^{r}$ forward mutations in wild- type and NER-defective rad14A cells. As shown in Figure 5A, neither the UV sensitivity nor the induction of UV mutagenesis is affected by the rev1S31A mutation in the SQ1 site when carried in the wild-type strain. However, the introduction of the rev1S31A mutant allele into the rad14s strain conferred a reduction in the frequency of UV-induced $\operatorname{can}^{r}$ mutations compared with that in the rad14A strain carrying the wild-type REV1 gene (Fig. 5B). Even though the rev1S31A mutation lowers the incidence of UV mutagenesis in the rad14A strain, it has no perceptible effect on the UV sensitivity of the rad14A strain (Fig. 5B). We infer from these observations that Rev1 phosphorylation contributes to an increase in the proficiency of Pol $\zeta$-mediated TLS, the need for which becomes more evident upon the inactivation of NER. Presumably, however, the increase in lesion bypass proficiency afforded by Rev1 phosphorylation is not substantial enough to confer a significant impact on UV resistance.

\section{Defects in replication checkpoint proteins affect Poly's proficiency in TLS via Rev1 phosphorylation}

Since the checkpoint proteins; the Mec1 kinase; Rad17, Mec3, and Ddc1, which form the checkpoint clamp; and $\operatorname{Rad} 24$, the clamp loader subunit, are all required for damage-induced Rev1 phosphorylation, and since the lack of Rev1 phosphorylation confers a reduction in UV mutagenesis in NER-defective cells, we surmised that the stimulatory effects of checkpoint proteins on UV mutagenesis that have been reported to occur in the absence of NER derive from their effects on Rev1 phosphorylation. If that were the case, then we would expect that the phosphorylation-defective rev1S31A mutation and the absence of checkpoint proteins that are required for Rev1 phosphorylation would affect UV mutagenesis in the rad14 $\Delta$ strain in a similar fashion and that they would exhibit an epistatic relationship in their effects on UV mutagenesis. To check the validity of this idea, we first compared the frequencies of UV-induced $\operatorname{can} 1^{r}$ mutations in the rad14s sml1s and rad14s sml1s mec1s strains. 
A.

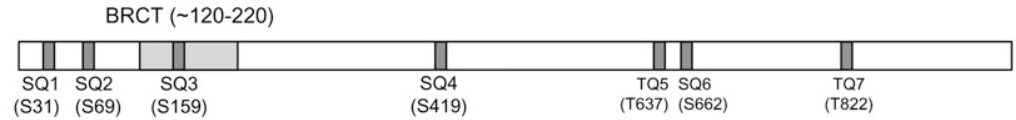

B.

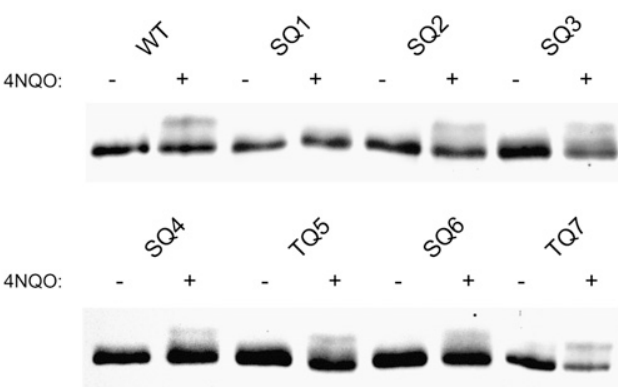

Figure 4. Identification of the Rev1 phosphorylation site. (A) Schematic map of seven potential (S/T)Q phosphorylation sites in Rev1. The position of the BRCT domain is also indicated. $(B)$ The effect of mutations in different potential phosphorylation sites (SQ or TQ) on Rev1 phosphorylation. Only the mutation in the SQ1 site $(\mathrm{S} 31 \rightarrow \mathrm{A})$ affects $4 \mathrm{NQO}-$ induced Rev1 phosphorylation. 4NQO-induced Rev1 phosphorylation was examined in yeast cells expressing the 4HA-tagged wild-type (WT) or mutant Rev1 protein. The $\mathrm{S}$ or $\mathrm{T}$ residue of $(\mathrm{S} / \mathrm{T}) \mathrm{Q}$ was changed to an alanine at each of the sites.
As shown in Figure 6A, a reduction in UV mutagenesis

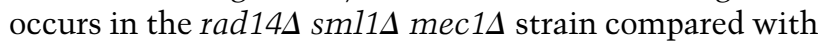
that in rad14 $\Delta$ sml1 $\Delta$ cells; however, the absence of Mec1 confers a greater reduction in UV mutagenesis in rad14A cells than does the rev1S31A mutation. Also, we find that the introduction of the rev1S31A mutation in the rad14A sml1s mec1s strain has no perceptible effect on UV mutagenesis or on UV sensitivity (Fig. 6A). These observations raise the possibility that in addition to affecting the proficiency of Pol $\zeta$-mediated TLS via Rev1 phosphorylation, the Mec1 kinase modulates phosphorylation of some other proteins that function with Pol $\zeta$.

Next, we examined whether the defects in checkpoint clamp and clamp loader subunits also affect UV mutagenesis similarly to the defects in Mecl kinase. A com-

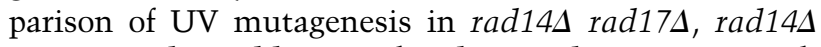

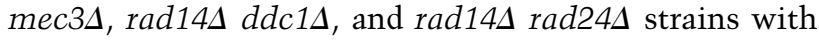
that in rad14A cells shows that a reduction in UV mutagenesis is incurred in rad14t cells in the absence of checkpoint clamp and clamp loader proteins (Fig. 6B). And, importantly, we find that the introduction of the rev1S31A mutation in rad14A cells lacking these checkpoint proteins confers no further change in UV mutation frequencies. Also, the UV sensitivity of rad14A cells lacking the checkpoint clamp or clamp loader proteins is not affected upon the introduction of rev1S31A mutation (Fig. 6B). However, similar to the effects of the mec1s mutation, the absence of checkpoint clamp and clamp loader proteins produces a greater defect in UV mutagenesis than that conferred by the rev1S31A mutation. We conclude from these observations that checkpoint proteins affect the proficiency of Pol $\zeta$-mediated TLS at least in part via their role in Rev1 phosphorylation, and surmise that they affect the phosphorylation of some other protein(s) involved in Pol $\zeta$-mediated TLS as well.

To identify the other protein factor(s) that, in addition to Rev1, could be the substrate for Mecl-mediated phosphorylation, we determined whether any of the proteins
A.

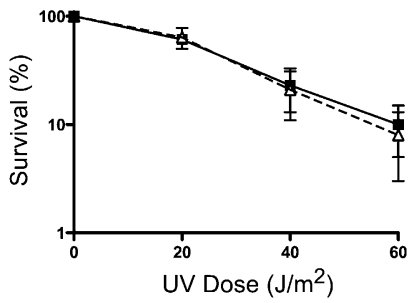

B.

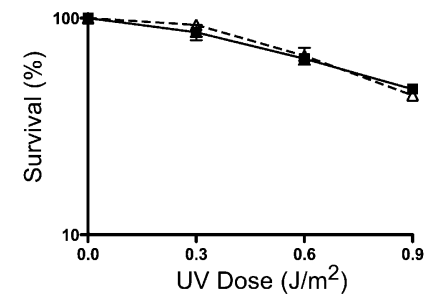

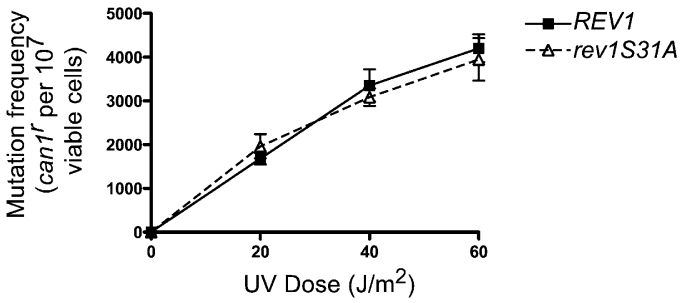

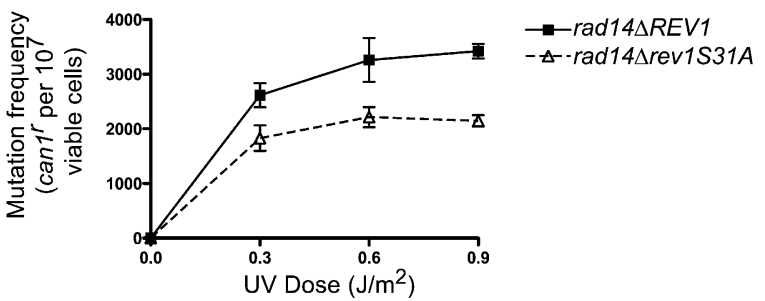

Figure 5. The phosphorylation-defective rev1S31A mutation affects the proficiency of UV mutagenesis in NER-defective yeast cells. UV survival and UV-induced can $1^{r}$ mutation frequencies were examined in rev1s cells carrying the wild-type (WT) or rev1S31A mutant gene on plasmid pVP21 in NER-proficient cells $(A)$ or NER-defective rad14 $\Delta$ cells $(B)$. The data represent averages of three independent experiments and error bars represent standard deviations. 
Pagès et al.

A.

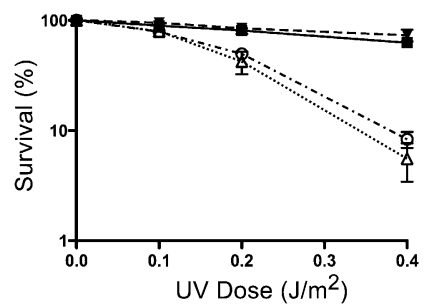

B.
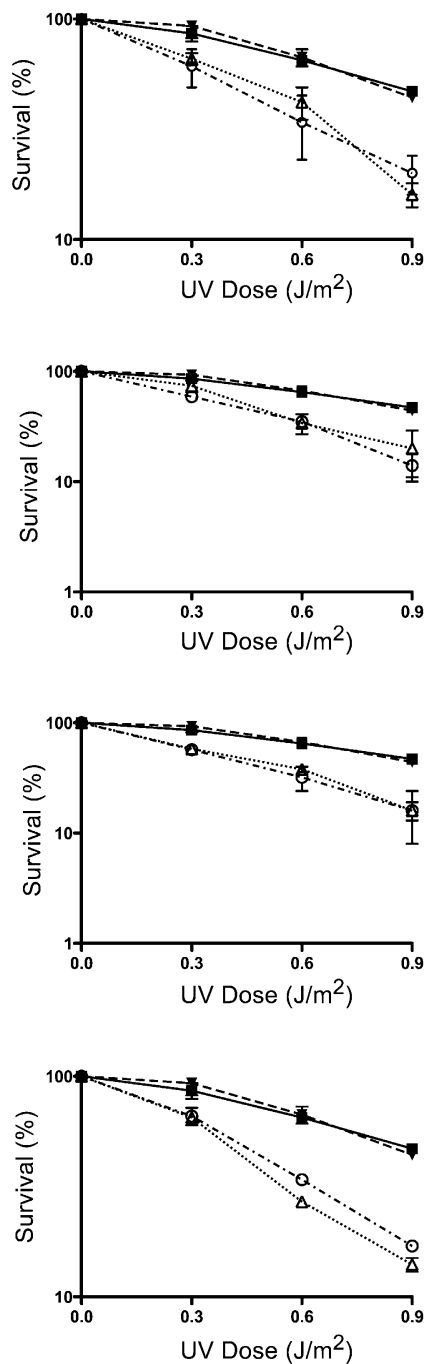

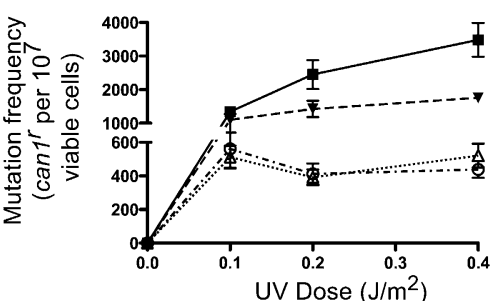

$-\operatorname{rad} 14 \triangle \mathrm{sm} / 1 \triangle \mathrm{REV} 1$

-7- $\operatorname{rad} 14 \Delta s m / 1 \Delta r e v 1 S 31 A$

$\cdots$ - $\operatorname{rad} 14 \Delta s m / 1 \Delta m e c 1 \Delta R E V 1$

-๑-. $\operatorname{rad} 14 \Delta \mathrm{sm} / 1 \Delta m e c 1 \Delta r e v 1 S 31 A$
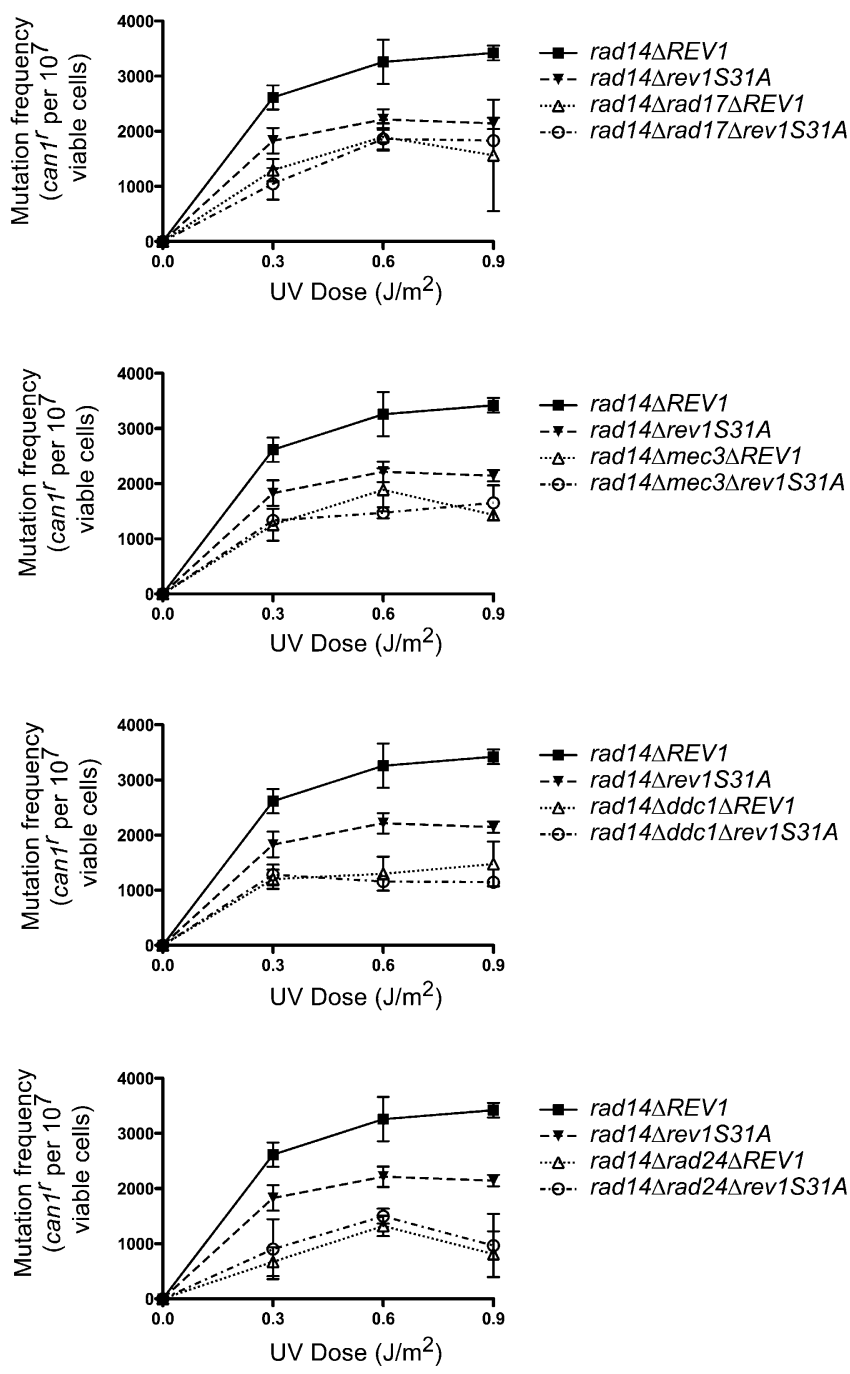

Figure 6. The effects of the rev1S31A mutation on UV mutagenesis in rad14A cells in the absence of checkpoint proteins. $(A)$ The mec1s mutation adversely affects UV mutagenesis in rad14A cells and it exhibits epistasis over rev1S31A mutation for UV mutagenesis in rad14 cells. (B) The effects of deletions of Rad17, Mec3, and Ddc1 subunits of checkpoint clamp and of the Rad24 subunit of checkpoint clamp loader on UV mutagenesis in rad14A cells are epistatic over effects of the rev1S31A mutation. UV-induced can $1^{r}$ mutation frequencies were examined in rad14A cells carrying genomic deletions of checkpoint genes and also harboring the genomic rev1A mutation, and carrying the wild-type REV1 or rev1S31A mutant gene on plasmid pVP21. The data represent averages of three independent experiments and error bars are standard deviations of these determinations.

that are known to function in Polל-dependent TLS are phosphorylated in response to DNA damage. Of the proteins required for Polל-dependent TLS, Rev3 contains seven potential (four SQ and three TQ) Mec1 phosphorylation sites spread throughout the protein, and Rev7 contains one SQ site toward its C terminus. In addition to the direct involvement of Pol $\zeta$ and Rev1 in Pol $\zeta$-dependent TLS, the Rad6-Rad18 enzyme complex plays a regulatory role in TLS mediated by Polל or Poln. In this enzyme complex, Rad6 contains one SQ site toward its $\mathrm{C}$ terminus and Rad18 contains two adjacent TQ, SQ sites in the middle of the protein. In addition, Pol32 is also 
required for Pol $\zeta$ function in TLS; however, it lacks any potential Mecl phosphorylation site. Poln functions in a pathway alternate to Polל for mediating TLS through UV-induced lesions, and it has two potential Mec1 phosphorylation sites: one SQ site in the middle and another SQ site toward the $C$ terminus of the protein.

The various C-terminally tagged proteins were expressed from their chromosomal loci in yeast cells and, using methods similar to those we used for Rev1 studies, the phosphorylation status of these TLS proteins was examined in yeast cells treated with 4-NQO (see the Supplemental Material; Supplemental Tables 4, 5). However, as shown in Supplemental Figure 2, we could detect no evidence for phosphorylation of Rev3, Rev7, $\operatorname{Rad} 30$, or Rad6 in undamaged or 4 NQO-damaged yeast cells. Since the expression level of genomic HA-tagged Rad18 was quite low and we were unable to establish its phosphorylation status, we examined whether mutational inactivation of the two potential Mecl phosphorylation sites present in Rad18 was detrimental to Polל-mediated TLS or to Poln function. However, in an NER-proficient yeast strain, UV survival or the frequency of UV-induced $\operatorname{can} 1^{r}$ mutations was not affected by the T282A-S284A double mutation made to inactivate the two potential TQ and SQ phosphorylation sites in the RAD18 gene (Supplemental Fig. 3A). Since the effect of the phosphorylationdefective mutation in the REV1 gene (rev1 S31A) became apparent only in the NER-defective rad14 $\Delta$ cells, we also determined the effects of the rad18 T282A-S284A mutation in rad14A cells. Here, also, we found that the UV survival and the frequency of UV-induced can $1^{r}$ muta-

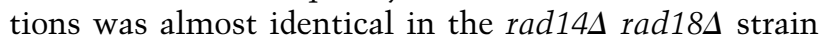
regardless of whether it carried the wild-type $R A D 18$ gene on plasmid pVP121 or the mutant rad18 gene on plasmid pVP124 (Supplemental Fig. 3B).

These observations lead us to conclude that Revl is unique among the known TLS factors in being regulated by Mec1-mediated phosphorylation, and that there still remains an as-yet-unidentified protein that functions in TLS and whose phosphorylation by Mec1 modulates TLS proficiency.

\section{Discussion}

The DNA damage-inducible replication checkpoint is dispensable for TLS in wild-type yeast cells

Based on genetic studies in yeast, the idea that checkpoint proteins, in particular the checkpoint clamp and clamp loader, are required for TLS has received considerable attention. In addition to the previous study (Paulovich et al. 1998) that suggested that, in S. cerevisiae, the components of checkpoint clamp and clamp loader were required for UV-induced mutagenesis and hence for Polל-mediated TLS, two other studies have implicated the involvement of these checkpoint proteins in TLS. In one of the studies, the Mec3 and Ddc1 checkpoint clamp subunits of $S$. cerevisiae were shown to interact physically with the Rev7 accessory subunit of Pol $\zeta$, and the loss of checkpoint clamp and clamp loader proteins was found to have an effect on the rate of Polל-dependent spontaneous mutagenesis of complex frameshifts (Sabbioneda et al. 2005). In another study, the Schizosaccharomyces pombe DinB TLS Pol was found to coprecipitate with the checkpoint clamp components, and the enhanced spontaneous mutagenesis that occurs in a Pol $\alpha$ mutant was shown to be reduced in the absence of checkpoint clamp loader subunit (Kai and Wang 2003).

In this study, we carry out a comprehensive analysis of the role of Mecl kinase and the subunits of checkpoint clamp and clamp loader on UV-induced mutagenesis. Since the genetic bases of UV mutagenesis is well established, and since it requires PCNA ubiquitination, the analysis of UV mutagenesis provides a useful measure of TLS. For spontaneous mutagenesis, such as the study of complex frameshifts in S. cerevisiae or that of the Pol $\alpha$ mutant in S. pombe (Kai and Wang 2003; Sabbioneda et al. 2005), it remains unclear whether these mutations result from TLS or whether they arise by some other means; as, for example, the elevated spontaneous mutability of the Pol $\alpha$ mutant could result from the role of $S$. pombe DinB in mismatch extension, as the related human Polк has been shown to carry out proficient mismatch extension (Washington et al. 2002). In that case, the primer terminus with a mispaired nucleotide resulting from the incorporation of a wrong nucleotide by the Pol $\alpha$ mutant could be extended by DinB. That could then suggest a role for checkpoint proteins in modulating DinB function in mismatch extension and not in TLS.

Our studies indicate that Mec1 kinase is not required for TLS carried out opposite UV lesions in wild-type yeast cells, and neither are the Rad17, Mec3, and Ddcl subunits of the checkpoint clamp or the Rad24 subunit of checkpoint clamp loader. Although the suggestion has been made for the direct involvement of checkpoint clamp in the binding of TLS Pols for mediating lesion bypass (for example, see Kai and Wang 2003; Sabbioneda et al. 2005), such a role is difficult to reconcile with the fact that Rad6-Rad18-mediated PCNA ubiquitination is essential for TLS and that the likely role of this PCNA modification is to facilitate the exchange of the replicative Pol with the TLS Pol (Zhuang et al. 2008). And, importantly, since the ability of a TLS Pol to bind PCNA is obligatory for its function in lesion bypass, that renders the possibility of a direct involvement of the checkpoint clamp in TLS implausible (Haracska et al. 2001; Acharya et al. 2008).

Although there is strong evidence that replication forks collapse in MMS-treated mec1s or rad53 $\Delta$ yeast cells (Tercero and Diffley 2001; Tercero et al. 2003), and that $\mathrm{Mec} 1$ and Rad53 are required to delay replication in UVirradiated rad144 cells (Neecke et al. 1999), it has remained unclear which of the various lesion bypass processes-TLS and/or template switching-depend on the activation of replication checkpoint for fork stabilization to occur. Our results indicating the lack of requirement of Mec1-mediated replication checkpoint for TLS could be explained if the functions of TLS Pols were closely coordinated with the replication ensemble, since that would then provide for relatively rapid and efficient 
means of lesion bypass. In that case, the stalling of the replication fork may not persist for a long enough period for checkpoint activation to occur. Alternatively, if the replicative Pols were to leave a gap opposite the DNA lesion resuming synthesis downstream, we would expect the persistence of gapped DNA to be sufficient to generate the signal necessary for checkpoint activation to occur; the ensuing delay in replication and in cell cycle progression would then provide the time interval needed for gap filling by TLS.

\section{Replication checkpoint proteins affect TLS proficiency in NER-defective yeast cells}

Our studies indicating a reduction in UV mutagenesis in rad14A cells in the absence of Mec1 kinase or the components of checkpoint clamp and clamp loader are in concert with previous studies in which similar results with the deletions of checkpoint clamp and clamp loader proteins were observed (Paulovich et al. 1998). Since UV lesions are not repaired in NER-defective cells, all of the unrepaired lesions have to be bypassed by TLS or by template switching; consequently, the frequency of UVinduced mutations is greatly enhanced in rad14s cells resulting from Pol $\zeta$-mediated TLS opposite DNA lesions.

The persistence of unrepaired UV lesions in rad14A cells causes the inhibition of S-phase progression dependent on Mec1 and Rad53 but not on the checkpoint clamp and clamp loader (Neecke et al. 1999). In the mec1 and rad53 mutants, progression through $\mathrm{S}$ phase in UVirradiated rad14t cells occurs from replication initiation from late origins. Presumably, in the absence of the Mec1mediated replication checkpoint in UV-irradiated rad14A cells, many of the replication forks collapse and unregulated DNA synthesis resumes from late firing origins (Neecke et al. 1999). Since the activation of the replication checkpoint in UV-irradiated rad14A cells requires the Mec1 kinase but UV mutagenesis can still occur in rad14A cells in the absence of Mec1 kinase, TLS in rad14A cells can transpire without the intervention of a replication checkpoint. The checkpoint proteins, Mec1, and the components of checkpoint clamp and clamp loader, however, modulate the proficiency of Pol $\zeta$-mediated TLS, at least in part through Rev1 phosphorylation, the need for which becomes evident in the absence of NER (see below).

\section{Checkpoint protein-mediated Rev1 phosphorylation modulates the efficiency of Poly function in UV mutagenesis}

Although Rev1 is a Y family Pol, its DNA polymerase activity is very specific for the insertion of a $\mathrm{C}$ opposite template G (Nelson et al. 1996a; Haracska et al. 2002), and Rev1 can promote replication through $\mathrm{N}^{2}$-dG minor groove DNA adducts by its proficient ability for inserting a C opposite them, from which another DNA Pol can then carry out the extension reaction (Nair et al. 2008). Even though opposite UV-induced DNA lesions Rev1's DNA polymerase activity plays no role in TLS, Rev1 functions as an indispensable structural element enabling
Polל to carry out its role in TLS opposite them. Rev1 physically interacts with the Rev3 catalytic subunit of Pol $\zeta$, and Revl binding enhances the proficiency of Pol $\zeta$ for extension from nucleotides inserted opposite a DNA lesion by another DNA Pol (Acharya et al. 2006).

The observations that damage-inducible Rev1 phosphorylation requires - in addition to Mec1/Ddc2 kinasethe components of checkpoint clamp and clamp loader, and that Rev1 phosphorylation occurs at the SQ1 Nterminal site, have allowed us to draw inferences as to how the effects of these checkpoint proteins on UV mutagenesis are related to their role in Rev1 phosphorylation. The results indicating that Rev1 phosphorylation positively modulates the proficiency of Pol $\zeta$-mediated TLS in NER-defective cells but not in NER-proficient cells, and that the checkpoint proteins involved in mediating Rev1 phosphorylation also affect TLS proficiency only in NER-defective cells, strongly suggest that checkpoint-mediated Rev1 phosphorylation contributes to the proficiency of Pol $\zeta$-mediated TLS. Furthermore, the epistasis of deletions of checkpoint proteins over the rev1S31A mutation for effects on UV mutagenesis implies that the checkpoint proteins contribute to increasing the proficiency of Pol $\zeta$-mediated TLS at least in part through Rev1 phosphorylation. Since deletions of checkpoint proteins generate a greater reduction in UV mutagenesis in rad14A cells than the rev1S31A mutation, that raises the possibility of checkpoint proteins additionally activating some other protein involved in Pol $\zeta$-mediated TLS by phosphorylating it, the identity of which remains to be determined.

Since both CPDs and (6-4) photoproducts would persist in DNA in NER-defective yeast cells and be subject to replicative bypass, it remains to be determined whether phosphorylated Rev1 is much more stimulatory to Polל's role in extending opposite from both types of DNA lesions than the unphosphorylated form, or whether this effect is more pronounced for a (6-4) photoproduct than for a CPD. Alternatively, or in addition, phosphorylated Rev1 may be more effective in complex formation with Pol $\zeta$ and its associated protein factors.

\section{Replication checkpoint, lesion bypass, and Rev1 phosphorylation}

Our observations that the checkpoint proteins, Mec1, and the components of checkpoint clamp and clamp loader are not essential for UV mutagenesis, but that checkpoint-induced Rev1 phosphorylation affects the proficiency of UV mutagenesis-which becomes evident in the absence of NER-pose the question as to where at the stalled fork might Rev1 phosphorylation occur in cells during replication. Since the inhibition of S-phase progression in UV-irradiated rad14A cells requires the Mec1 and Rad53 kinases and not the proteins of the checkpoint clamp and clamp loader (Neecke et al. 1999), whereas Rev1 phosphorylation requires the Mecl kinase and the checkpoint clamp and clamp loader but not the Rad53 kinase, we infer that Rev1 phosphorylation can occur in the absence of replication checkpoint. The 
transient stalling of the replication fork at the lesion site could generate a signal for the loading of checkpoint clamp by the clamp loader on the gapped DNA on the lagging strand, following which the Mec1/Ddc2 kinase would bind the clamp, phosphorylating Rev1 and other proteins present in the replication ensemble. According to this scenario, Rev1 phosphorylation might occur after Rev1 and the other proteins involved in Pol $\zeta$-mediated TLS have been assembled at the site of stalled replication fork. Further, we surmise that, although the transient stalling of the replication fork that ensues during TLS may be adequate for activating Rev1 phosphorylation, it does not produce the threshold level of signal needed for checkpoint activation to occur.

Another question to consider is which of the lesion bypass processes become inoperative in the absence of damage-induced replication checkpoint. Since lesion bypass in the absence of TLS occurs by Rad5-, Mms2-, and Ubc13-dependent (Torres-Ramos et al. 2002; Gangavarapu et al. 2006; Blastyak et al. 2007) or by Rad51-, Rad52-, and Rad54-dependent (Prakash 1981; Gangavarapu et al. 2007) template-switching pathways, the possibility remains that these processes require the establishment of a replication checkpoint to ensure that the integrity of the replication fork stalled at the lesion site is maintained. Such a possibility is suggested also from the greatly elevated UV sensitivity and enhanced UV mutagenesis of mec1s rad30s cells, as that could result from the inhibition of Rad5-, Mms2-, Ubc13-, Rad51-, Rad52-, and Rad54-dependent template-switching pathways in the absence of Mecl. Moreover, the greatly enhanced UV sensitivity of the mec1s pol30-119 double mutant over that of mec1s or pol30-119 mutants is in line with a role for the Mec1-mediated replication checkpoint in Rad51-, Rad52-, and Rad54-mediated lesion bypass by recombinational means.

\section{Conclusions}

This study shows that the Mecl kinase and the components of checkpoint clamp and clamp loader are not required for TLS in yeast cells, and we infer from our results that the intervention of the replication checkpoint is not needed for TLS to occur. The replication checkpoint proteins, however, modulate the proficiency of Pol $\zeta$-mediated TLS at least in part by effecting Rev1 phosphorylation. The dependence of Pol $\zeta$ 's ability to carry out efficient TLS on checkpoint proteins as well as on Rev1 phosphorylation can be discerned in rad14s cells, where the lesion load is greatly accentuated because of the absence of NER, and therefore the need for more proficient TLS becomes more acute.

\section{Materials and methods}

\section{Yeast strains}

All yeast strains are derived from DBY747 (MATa his3-41 leu2-3 leu2-112 ura3-52) or from the isogenic EMY74.7 (MATa his3-41 leu2-3 leu2-112 trp1s ura3-52) strain. Genomic deletions of checkpoint genes were made in the wild-type yeast strain
EMY74.7 (Supplemental Table 1). For Rev1 phosphorylation assays, the 4-HA-tagged wild-type Rev1 or mutant Rev1 was expressed from a YCplac111-based plasmid (Supplemental Fig. 1) in different yeast strains (Supplemental Table 2). UV-induced mutagenesis studies for examining the effects of the phosphorylationdefective rev1S31A mutation were carried out in yeast strains in which the chromosomal copy of REV1 has been deleted, and the wild-type Rev1 or the Rev1 S31A mutant protein was expressed from the YCplac111-based plasmid (Supplemental Table 3).

\section{Plasmids for Rev1 expression}

The REV1 ORF plus 1050 nucleotides (nt) of its upstream region that include the REV1 promoter and $500 \mathrm{nt}$ of the downstream region that include the REV1 transcription terminator was cloned into the vector YCplac111 (pTB364) (Gietz and Sugino 1988), which carries the LEU2 gene, the autonomous replicating sequence $A R S 1$, and the centromeric CEN4 region (Supplemental Fig. 1A), generating plasmid pVP21.

A BglII restriction site was added by site-directed mutagenesis right before the stop codon of REV1. A 33-mer oligonucleotide duplex containing the HA-coding sequence and a BamHI and a BglII restriction site $\left(5^{\prime}\right.$-GATCATTACCCGTATGATGTTCCG GATTACGCA-3'/5'-GATCTGCGTAATCCGGAACATCATAC GGGTAAT-3') was inserted into the BglII restriction site, adding an HA tag in fusion with REV1, and regenerating a unique BglII restriction site (Supplemental Fig. 1B). This step was repeated three times in order to get four HA tags in fusion with REV1, generating plasmid pVP64 (Supplemental Fig. 1A).

Rev1 has seven (S/T)Q sites that are known to be the target of PI-3 protein kinases such as Mec1 (Fig. 4A). We individually inactivated these seven potential phosphorylation sites by sitedirected mutagenesis by changing the serine or threonine residue to an alanine: S31 $\rightarrow$ A (SQ1), S69 $\rightarrow$ A (SQ2), S159 $\rightarrow$ A (SQ3), $\mathrm{S} 419 \rightarrow \mathrm{A}(\mathrm{SQ} 4), \mathrm{T} 637 \rightarrow \mathrm{A}(\mathrm{TQ} 5), \mathrm{S} 662 \rightarrow \mathrm{A}(\mathrm{SQ} 6)$, and T822 $\rightarrow$ A (TQ7). Each of these mutations was introduced into plasmid pVP64, leading to an HA-tagged version of these mutant proteins.

\section{Analysis of damage-induced Rev1 phosphorylation}

An overnight culture of cells expressing 4HA-tagged wild-type or mutationally altered Rev1 at (S/T)Q sites (Supplemental Table 2) from plasmid pVP64 (Supplemental Fig. 1) was grown in synthetic complete (SC) medium lacking leucine (SC - leu) and used to inoculate $50 \mathrm{~mL}$ of yeast extract-peptone-dextrose (YPD) media. Once the cells had reached exponential phase $\left(\sim 10^{7}\right.$ cells per milliliter), $25 \mathrm{~mL}$ of the culture were treated with $4 \mathrm{NQO}$ $(2 \mu \mathrm{g} / \mathrm{mL})$ for $15 \mathrm{~min}$, and $25 \mathrm{~mL}$ remained untreated. Cells were then washed once with water and once with $1 \times$ PBS, $10 \%$ glycerol, and $1 \mathrm{mM}$ EDTA before protein extraction. Cells were broken in a bead beater with $3 \mathrm{vol}$ of $1 \times \mathrm{PBS}+10 \%$ glycerol + protease inhibitors + phosphatase inhibitor and 2 vol of zirconia/ silica beads. Cell debris was removed by centrifugation, and total protein concentration was determined using the Bio-Rad protein assay.

Seventy-five micrograms of the crude protein extract were loaded on a $6 \%$ acrylamide/bisacrylamide $(77: 1)$ gel. The combination of this low cross-linking percentage with a long migration of the gel (15 cm for the $100-\mathrm{kDa}$ marker) improved the resolution of the phospho-forms of Rev1. Rev1 was detected by Western blotting with anti-HA antibody (Abcam, ab9110).

\section{UV survival and mutagenesis}

To examine the effects of checkpoint mutations, the wild-type and isogenic derivatives of genomic deletions of checkpoint 
genes (Supplemental Table 1) were grown to logarithmic phase in SC medium supplemented with 10 amino acids plus adenine and uracil $(\mathrm{SC}+12)$. Cultures were washed by centrifugation, followed by sonication to disperse cell clumps. After sonication, the cell suspension was centrifuged again and resuspended to a density of $\sim 2 \times 10^{8}$ cells per milliliter. Cells were diluted, and serial dilutions were spread on SC +12 plates for viability determinations and on $\mathrm{SC}+12$ plates lacking arginine but containing canavanine (SC - arg + can) for determination of $C A N 1^{S}$ to can $1^{r}$ mutation frequencies. The plates were UV-irradiated and incubated in the dark for $5 \mathrm{~d}$ prior to counting of colonies. The effects of the rev1S31A mutation on UV mutagenesis were examined in rev1s cells carrying the wild-type REV1 gene or the rev1S31A mutant gene on plasmid pVP21 (Supplemental Table 3). To maintain selection for the plasmid, yeast strains were grown in SC - leu and UV survival and the frequency of UV-induced $\operatorname{can} 1^{r}$ mutations were assessed by plating cells on SC - leu and SC - leu - arg + can media, respectively.

\section{Acknowledgments}

This work was supported by grant CA107650 from the National Cancer Institute, National Institutes of Health.

\section{References}

Acharya N, Johnson RE, Prakash S, Prakash L. 2006. Complex formation with Rev1 enhances the proficiency of yeast DNA polymerase $\zeta$ for mismatch extension and for extension opposite from DNA lesions. Mol Cell Biol 26: 9555-9563.

Acharya N, Yoon J-H, Gali H, Unk I, Haracska L, Johnson RE, Hurwitz J, Prakash L, Prakash S. 2008. Roles of PCNAbinding and ubiquitin-binding domains in human DNA polymerase $\eta$ in translesion DNA synthesis. Proc Natl Acad Sci 105: 17724-17729.

Blastyak A, Pinter L, Unk I, Prakash L, Prakash S, Haracksa L. 2007. Yeast Rad5 protein required for postreplication repair has a DNA helicase activity specific for replication fork regression. Mol Cell 28: 167-175.

Bresson A, Fuchs RPP. 2002. Lesion bypass in yeast cells: Pol $\eta$ participates in a multi-DNA polymerase process. EMBO I 21: 3881-3887.

Cobb JA, Bjergbaek L, Shimada K, Frei C, Gasser SM. 2003. DNA polymerase stabilization at stalled replication forks requires Mec1 and the RecQ helicase Sgs1. EMBO I 22: 4325-4336.

Cobb JA, Schleker T, Rojas V, Bjergbaek L, Tercero JA, Gasser SM. 2005. Replisome instability, fork collapse, and gross chromosomal rearrangements arise synergistically from Mecl kinase and RecQ helicase mutations. Genes \& Dev 19: 3055-3069.

Gangavarapu V, Haracska L, Unk I, Johnson RE, Prakash S, Prakash L. 2006. Mms2-Ubc13-dependent and -independent roles of Rad5 ubiquitin ligase in postreplication repair and translesion DNA synthesis in Saccharomyces cerevisiae. Mol Cell Biol 26: 7783-7790.

Gangavarapu V, Prakash S, Prakash L. 2007. Requirement of RAD52 group genes for post-replication repair of UV-damaged DNA in Saccharomyces cerevisiae. Mol Cell Biol 27: 77587764.

Gietz RD, Sugino A. 1988. New yeast-Escherichia coli shuttle vectors constructed with in vitro mutagenized yeast genes lacking six-base pair restriction sites. Gene 74: 527534.

Haracska L, Kondratick CM, Unk I, Prakash S, Prakash L. 2001. Interaction with PCNA is essential for yeast DNA polymerase $\eta$ function. Mol Cell 8: 407-415.
Haracska L, Prakash S, Prakash L. 2002. Yeast Rev1 protein is a G template-specific DNA polymerase. I Biol Chem 277: 15546-15551.

Haracska L, Torres-Ramos CA, Johnson RE, Prakash S, Prakash L. 2004. Opposing effects of ubiquitin conjugation and SUMO modification of PCNA on replicational bypass of DNA lesions in Saccharomyces cerevisiae. Mol Cell Biol 24: 4267-4274.

Hoege C, Pfander B, Moldovan G-L, Pyrowolakis G, Jentsch S. 2002. RAD6-dependent DNA repair is linked to modification of PCNA by ubiquitin and SUMO. Nature 419: 135-141.

Johnson RE, Prakash S, Prakash L. 1999. Efficient bypass of a thymine-thymine dimer by yeast DNA polymerase, Pol $\eta$. Science 283: 1001-1004.

Johnson RE, Washington MT, Haracska L, Prakash S, Prakash L. 2000a. Eukaryotic polymerases $\iota$ and $\zeta$ act sequentially to bypass DNA lesions. Nature 406: 1015-1019.

Johnson RE, Washington MT, Prakash S, Prakash L. 2000b. Fidelity of human DNA polymerase $\eta$. I Biol Chem 275: 7447-7450.

Kai M, Wang TS. 2003. Checkpoint activation regulates mutagenic translesion synthesis. Genes \& Dev 17: 64-76.

Majka J, Burgers PMJ. 2003. Yeast Rad17/Mec3/Ddc1: A sliding clamp for the DNA damage checkpoint. Proc Natl Acad Sci 100: 2249-2254.

McDonald JP, Levine AS, Woodgate R. 1997. The Saccharomyces cerevisiae RAD30 gene, a homologue of Escherichia coli $\operatorname{din} B$ and umuC, is DNA damage inducible and functions in a novel error-free postreplication repair mechanism. Genetics 147: 1557-1568.

Nair DT, Johnson RE, Prakash L, Prakash S, Aggarwal AK. 2008. Protein-template directed synthesis across an acroleinderived DNA adduct by yeast Rev1 DNA polymerase. Structure 16: 239-245.

Neecke H, Lucchini G, Longhese MP. 1999. Cell cycle progression in the presence of irreparable DNA damage is controlled by a Mec1- and Rad53-dependent checkpoint in budding yeast. $E M B O J$ 18: 4485-4497.

Nelson JR, Lawrence CW, Hinkle DC. 1996a. Deoxycytidyl transferase activity of yeast REV1 protein. Nature 382: 729-731.

Nelson JR, Lawrence CW, Hinkle DC. 1996b. Thymine-thymine dimer bypass by yeast DNA polymerase $\zeta$. Science 272: 1646-1649.

Nyberg KA, Michelson RJ, Putnam CW, Weinert TA. 2002. Toward maintaining the genome: DNA damage and replication checkpoints. Annu Rev Genet 36: 617-656.

Paulovich AG, Armour CD, Hartwell LH. 1998. The Saccharomyces cerevisiae RAD9, RAD17, RAD24, and MEC3 genes are required for tolerating irreparable, ultraviolet-induced DNA damage. Genetics 150: 75-93.

Paulsen RD, Cimprich KA. 2007. The ATR pathway: Finetuning the fork. DNA Repair (Amst) 6: 953-966.

Prakash L. 1981. Characterization of postreplication repair in Saccharomyces cerevisiae and effects of rad6, rad18, rev3 and rad52 mutations. Mol Gen Genet 184: 471-478.

Prakash S, Johnson RE, Prakash L. 2005. Eukaryotic translesion synthesis DNA polymerases: Specificity of structure and function. Annu Rev Biochem 74: 317-353.

Sabbioneda S, Minesinger BK, Giannattasio M, Plevani P, MuziFalconi M, Jinks-Robertson S. 2005. The 9-1-1 checkpoint clamp physically interacts with Pol $\zeta$ and is partially required for spontaneous Pol $\zeta$-dependent mutagenesis in Saccharomyces cerevisiae. J Biol Chem 280: 38657-38665.

Sabbioneda S, Bortolomai I, Giannattasio M, Plevani P, MuziFalconi M. 2007. Yeast Rev1 is cell cycle regulated, phos- 
phorylated in response to DNA damage and its binding to chromosomes is dependent upon MEC1. DNA Repair (Amst) 6: $121-127$.

Sancar A. 1996. DNA excision repair. Annu Rev Biochem 65: 43-81.

Sogo JM, Lopes M, Foiani M. 2002. Fork reversal and ssDNA accumulation at stalled replication forks owing to checkpoint defects. Science 297: 599-602.

Stelter P, Ulrich HD. 2003. Control of spontaneous and damageinduced mutagenesis by SUMO and ubiquitin conjugation. Nature 425: 188-191.

Tercero JA, Diffley JF. 2001. Regulation of DNA replication fork progression through damaged DNA by the Mec1/Rad53 checkpoint. Nature 412: 553-557.

Tercero JA, Longhese MP, Diffley JFX. 2003. A central role for DNA replication forks in checkpoint activation and response. Mol Cell 11: 1323-1336.

Torres-Ramos C, Prakash S, Prakash L. 2002. Requirement of $R A D 5$ and MMS2 for post replication repair of UV-damaged DNA in Saccharomyces cerevisiae. Mol Cell Biol 22: 24192426.

Washington MT, Johnson RE, Prakash S, Prakash L. 2000. Accuracy of thymine-thymine dimer bypass by Saccharomyces cerevisiae DNA polymerase $\eta$. Proc Natl Acad Sci 97: 3094-3099.

Washington MT, Johnson RE, Prakash L, Prakash S. 2002. Human DINB1-encoded DNA polymerase $\kappa$ is a promiscuous extender of mispaired primer termini. Proc Natl Acad Sci 99: 1910-1914.

Washington MT, Prakash L, Prakash S. 2003. Mechanism of nucleotide incorporation opposite a thymine-thymine dimer by yeast DNA polymerase $\eta$. Proc Natl Acad Sci 100: 1209312098.

You Y-H, Lee D-H, Yoon J-H, Nakajima S, Yasui A, Pfeifer GP. 2001. Cyclobutane pyrimidine dimers are responsible for the vast majority of mutations induced by UVB irradiation in mammalian cells. J Biol Chem 276: 44688-44694.

Yu S-L, Johnson RE, Prakash S, Prakash L. 2001. Requirement of DNA polymerase $\eta$ for error-free bypass of UV-induced CC and TC photoproducts. Mol Cell Biol 21: 185-188.

Zhuang Z, Johnson RE, Haracksa L, Prakash L, Prakash S, Benkovic SJ. 2008. Regulation of polymerase exchange be-

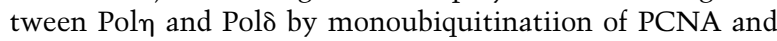
the movement of DNA polymerase holoenzyme. Proc Natl Acad Sci 105: 5361-5366. 


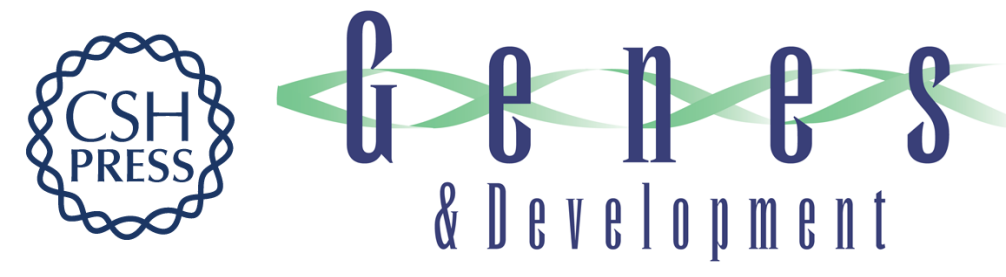

\section{Role of DNA damage-induced replication checkpoint in promoting lesion bypass by translesion synthesis in yeast}

Vincent Pagès, Sergio R. Santa Maria, Louise Prakash, et al.

Genes Dev. 2009, 23:

Access the most recent version at doi:10.1101/gad.1793409

Supplemental http://genesdev.cshlp.org/content/suppl/2009/06/01/23.12.1438.DC1
Material

References This article cites 42 articles, 26 of which can be accessed free at:

http://genesdev.cshlp.org/content/23/12/1438.full.html\#ref-list-1

License

Email Alerting Receive free email alerts when new articles cite this article - sign up in the box at the top

Service right corner of the article or click here.

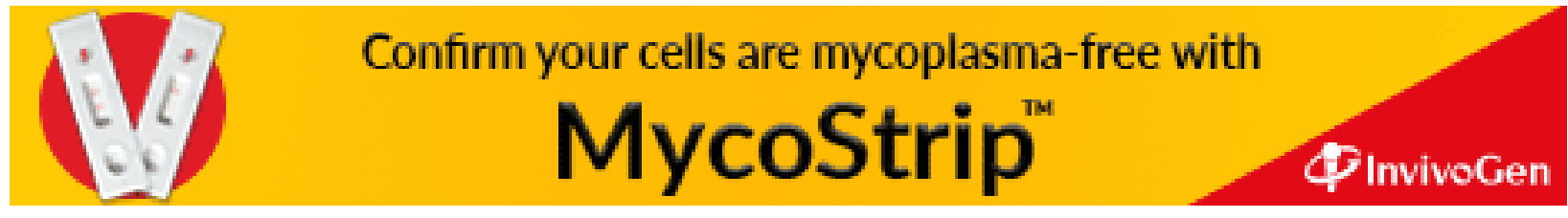

